\title{
A reading guide for last passage times with financial applications in view
}

\author{
Nikeghbali, Ashkan ; Platen, Eckhard
}

\begin{abstract}
In this survey on last passage times, we propose a new viewpoint which provides a unified approach to many different results which appear in the mathematical finance literature and in the theory of stochastic processes. In particular, we are able to improve the assumptions under which some well-known results are usually stated. Moreover we give some new and detailed calculations for the computation of the distribution of some large classes of last passage times. We have kept in this survey only the aspects of the theory which we expect potentially to be relevant for financial applications.
\end{abstract}

DOI: https://doi.org/10.1007/s00780-013-0207-6

Posted at the Zurich Open Repository and Archive, University of Zurich ZORA URL: https://doi.org/10.5167/uzh-86956

Journal Article

Published Version

Originally published at:

Nikeghbali, Ashkan; Platen, Eckhard (2013). A reading guide for last passage times with financial applications in view. Finance and Stochastics, 17(3):615-640.

DOI: https://doi.org/10.1007/s00780-013-0207-6 


\title{
A reading guide for last passage times with financial applications in view
}

\author{
Ashkan Nikeghbali · Eckhard Platen
}

Received: 6 August 2008 / Accepted: 11 May 2012 / Published online: 23 May 2013

(C) Springer-Verlag Berlin Heidelberg 2013

\begin{abstract}
In this survey on last passage times, we propose a new viewpoint which provides a unified approach to many different results which appear in the mathematical finance literature and in the theory of stochastic processes. In particular, we are able to improve the assumptions under which some well-known results are usually stated. Moreover we give some new and detailed calculations for the computation of the distribution of some large classes of last passage times. We have kept in this survey only the aspects of the theory which we expect potentially to be relevant for financial applications.
\end{abstract}

Keywords Last passage times $\cdot$ Class $\Sigma \cdot$ Running maximum

Mathematics Subject Classification (2000) 60G17 · 62P05

JEL Classification $\mathrm{C} 02 \cdot \mathrm{C} 10 \cdot \mathrm{G} 10$

Notation In this paper, $\left(\Omega, \mathcal{F},\left(\mathcal{F}_{t}\right)_{t \geq 0}, \mathbb{P}\right)$ will denote a filtered probability space. $\mathcal{C}\left(\mathbb{R}_{+}, \mathbb{R}\right)$ is the space of continuous functions from $\mathbb{R}_{+}$to $\mathbb{R} . \mathcal{D}\left(\mathbb{R}_{+}, \mathbb{R}\right)$ is the space of càdlàg functions from $\mathbb{R}_{+}$to $\mathbb{R}$. If $A=\left(A_{t}\right)_{t \geq 0}$ is an increasing process, we set $A_{\infty}:=\lim _{t \rightarrow \infty} A_{t}$.

A. Nikeghbali $(\bowtie)$

Institut für Mathematik, Universität Zürich, Winterthurerstrasse 190, 8057 Zürich, Switzerland e-mail: ashkan.nikeghbali@math.uzh.ch

A. Nikeghbali

Department of Banking and Finance, Universität Zürich, Plattenstrasse 14, 8032 Zürich, Switzerland

E. Platen

Finance Discipline Group and School of Mathematical Sciences, University of Technology Sydney, PO Box 123, Broadway, NSW 2007, Australia

e-mail: eckhard.platen@uts.edu.au 
If $X=\left(X_{t}\right)_{t \geq 0}$ is a stochastic process, then $\bar{X}_{t}$ denotes the running maximum $\sup _{u \leq t} X_{u}$. We also recall that a stochastic process $X$ is said to be of class $(D)$ if the family of random variables $\left\{\left|X_{T}\right| \mathbb{1}_{\{T<\infty\}}: T\right.$ a stopping time $\}$ is uniformly integrable.

We say that a nonnegative local martingale $M=\left(M_{t}\right)_{t \geq 0}$ belongs to $\mathcal{M}_{0}$ if $\left(\bar{M}_{t}\right)_{t \geq 0}$ is continuous and $M_{0}=1, \lim _{t \rightarrow \infty} M_{t}=0$.

We say that $g$ is the end of a predictable or an optional set if

$$
g=\sup \{t:(t, \omega) \in \Gamma\}
$$

where $\Gamma$ is a predictable or an optional set, respectively.

\section{Introduction}

Ends of optional sets, most commonly called "last passage times", are random times which are not stopping times. For instance, if $\left(W_{t}\right)_{t \geq 0}$ is a standard Brownian motion, then

$$
g=\sup \left\{t \leq 1: W_{t}=0\right\}
$$

is such a time. The distribution of this time was already studied by Paul Lévy in [19]. Another interesting example, which will play an important role in our discussion, can be constructed as follows. Let $M$ be a continuous nonnegative local martingale with $M_{0}=1$ and $\lim _{t \rightarrow \infty} M_{t}=0$. Then

$$
g=\sup \left\{t \geq 0: M_{t}=\bar{M}_{t}\right\}
$$

is another such time. As illustrated with the above two examples, last passage times look into the future and that feature makes their analysis more delicate. The standard theorems from martingale theory such as Doob's optional stopping theorem do not apply to them. They have nevertheless received some attention in stochastic analysis (see e.g. the papers by Chung [8] and Azéma [1]). They play an important role in the theory of enlargements of filtrations (see e.g. the works by Barlow [4], Jeulin [16], Yor [42], Nikeghbali and Yor [32], or the monograph [11] for a survey), in the characterisations of strong Brownian filtrations (see the monograph by Mansuy and Yor [23] for details and references), and in path decompositions of diffusions (e.g. [16] and [30]). One should mention that last passage times have also inspired some fear because the standard martingale techniques do not apply to them, as noticed by Kai Lai Chung [8]:

For some reason the notion of a last exit time, which is manifestly involved in the arguments, would not be dealt with openly and directly. This may be partially due to the fact that such a time is not an "optional" (or "stopping") time, does not belong to the standard equipment, and so must be evaded at all costs.

Last passage times have also played an increasing role in financial modelling in recent years, thus outlining the need for a framework which would allow a systematic study of them. They appear, for instance, in the seminal paper [13] of Elliott, 
Jeanblanc and Yor on models of default risk (see also the recent book [15] for more references towards this direction), or in the work by Imkeller [14] on insider trading. In these works, examples of last passage times such as the last time some transient diffusion hits some fixed level, or the last time the standard Brownian motion hits zero before some fixed time, are considered; they do not contain statements which would hold for any such time. On the other hand, in a series of very recent papers (which have grown into the book [39]), Madan, Roynette and Yor in [21, 22] have discovered that the prices of European put and call options for asset prices which can be modelled by nonnegative and continuous martingales that vanish at infinity, can be expressed in terms of the probability distributions of some last passage times. Their formulae are very general and exhibit the striking feature of being model independent. More precisely, let $M$ be a continuous and nonnegative local martingale with $M_{0}=1$ and $\lim _{t \rightarrow \infty} M_{t}=0$. Then Madan, Roynette and Yor prove that the price of a European put option on a risky asset modelled by $M$, with strike $K \geq 0$, is given by

$$
\mathbb{E}\left[\left(K-M_{t}\right)^{+}\right]=K \mathbb{P}\left[g_{K} \leq t\right],
$$

where

$$
g_{K}=\sup \left\{s: M_{s}=K\right\} .
$$

The above representation holds for instance if the underlying filtered probability space $\left(\Omega, \mathcal{F},\left(\mathcal{F}_{t}\right)_{t \geq 0}, \mathbb{P}\right)$ satisfies the usual assumptions, which are standard in the mathematical finance literature. A formula similar to (1.1) holds as well for call options, but one needs to make some extra assumptions ([39], Chap. 2):

- the martingale $M$ must be a true martingale, i.e., $\mathbb{E}\left[M_{t}\right]=1$ for all $t \geq 0$;

- the filtration $\left(\mathcal{F}_{t}\right)_{t \geq 0}$ is the (raw) filtration generated by $M$ and made rightcontinuous, and $\mathcal{F}=\mathcal{F}_{\infty}$.

If the above conditions hold, then it is shown in [39] that the price of the European call option associated to $M$ and $K \geq 0$ is given by

$$
\mathbb{E}\left[\left(M_{t}-K\right)^{+}\right]=\mathbb{P}^{(M)}\left[g_{K} \leq t\right],
$$

where $g_{K}$ is defined by (1.2) and $\mathbb{P}^{(M)}$ is the unique probability measure on $\left(\Omega, \mathcal{F}_{\infty}\right)$ satisfying

$$
\mathbb{P}_{\mid \mathcal{F}_{t}}^{(M)}=M_{t} . \mathbb{P}_{\mid \mathcal{F}_{t}} .
$$

In fact, formula (1.3) suggests a natural approach to understand last passage times, but it also reveals all the difficulties that can be attached to such times. Indeed in the framework suggested in [39] and recalled above, formula (1.3) is not rigorously correct. There are two issues that can fortunately be fixed:

- The measure $\mathbb{P}^{(M)}$ does not always exist; one has to make extra assumptions of topological nature on the filtration $\left(\mathcal{F}_{t}\right)_{t \geq 0}$. These conditions were introduced by Parthasarathy in his book [33] and we call them (P). We have included them in the Appendix in order to concentrate exclusively on last passage times in the main body of the paper. An important fact for now is that the canonical path 
spaces $\mathcal{C}\left(\mathbb{R}_{+}, \mathbb{R}\right)$ and $\mathcal{D}\left(\mathbb{R}_{+}, \mathbb{R}\right)$, endowed with the filtration $\mathcal{F}_{t}=\sigma\left(X_{s}, s \leq t\right)$ and $\mathcal{F}_{\infty}=\sigma\left(X_{s}, s \geq 0\right)$, where $\left(X_{t}\right)_{t \geq 0}$ is the coordinate process, satisfy condition $(\mathrm{P})$ and consequently the measure $\mathbb{P}^{(M)}$ exists.

- The other problem is caused by the fact that if one takes the usual augmentation of the Wiener space with respect to Wiener measure, then the measure $\mathbb{P}^{(M)}$ does not exist again (see the introduction of [24]). This is due to the fact that since $\lim _{t \rightarrow \infty} M_{t}=0$, the measure $\mathbb{P}^{(M)}$ is locally absolutely continuous with respect to Wiener measure but is globally singular with respect to it, and putting all the negligible sets in $\mathcal{F}_{0}$ prevents such a measure from existing. On the other hand, without the usual assumptions, many results from the theory of stochastic processes do not hold or hold only almost surely at best (e.g. the existence of an adapted, rightcontinuous with left limits version for martingales, the Doob-Meyer decomposition, the different projections, stochastic integrals, etc.). For instance, it is shown in [24] that without the usual augmentation on the Wiener space, there does not exist an adapted and continuous version of the local time which is defined everywhere. More generally it is well known that one needs some sort of augmentation to have well-defined versions of continuous and increasing processes which are finite for finite times and which are adapted. To overcome this difficulty, which can become very annoying, it is proposed in [24] to consider a new kind of augmentation of filtrations, called the natural augmentation (in fact this augmentation has already been discovered earlier by Bichteler in [5]). We have also stated a few definitions and results related to this augmentation in the Appendix. It is enough for our purpose to note that a filtered probability space $\left(\Omega, \mathcal{F},\left(\mathcal{F}_{t}\right)_{t \geq 0}, \mathbb{P}\right)$ satisfies the natural conditions if the filtration $\left(\mathcal{F}_{t}\right)_{t \geq 0}$ is right-continuous and for all $t \geq 0$ and for every $\mathbb{P}$-negligible set $A \in \mathcal{F}_{t}$, all the subsets of $A$ are contained in $\mathcal{F}_{0}$. This definition excludes events which are negligible in $\mathcal{F}_{\infty}$ but not negligible for any $\mathcal{F}_{t}$ (for instance $\{g>1\}$, where $g=\sup \left\{t: W_{t}=0\right\}$ and $W$ is Brownian motion). Moreover most of the important results of the theory of stochastic processes which are proved under the usual conditions also hold under the natural conditions. The remarkable feature of this natural augmentation is that it combines very well with changes of probability measures which are only locally absolutely continuous. More precisely a filtered probability space $\left(\Omega, \mathcal{F},\left(\mathcal{F}_{t}\right)_{t \geq 0}, \mathbb{P}\right)$ is said to satisfy the property (NP) if and only if it is the natural enlargement of a filtered probability space $\left(\Omega, \mathcal{F}^{0},\left(\mathcal{F}_{t}^{0}\right)_{t \geq 0}, \mathbb{P}^{0}\right)$ such that the filtered measurable space $\left(\Omega, \mathcal{F}^{0},\left(\mathcal{F}_{t}^{0}\right)_{t \geq 0}\right)$ enjoys property (P) explained in the appendix. The measure $\mathbb{P}^{(M)}$ always exists under the property (NP) which is hence the condition to establish formula (1.3).

Both (1.1) and (1.3) suggest that the processes $(K-M)^{+}$and $(M-K)^{+}$are uniquely characterised by a pair $(\mathcal{Q}, g)$, where $\mathcal{Q}$ is a measure and $g$ is the last zero of the process. In the first case $\mathcal{Q}=K . \mathbb{P}$, which is equivalent to $\mathbb{P}$, and in the second case $\mathcal{Q}=\mathbb{P}^{(M)}$ is singular with respect to $\mathbb{P}$. We present an approach to last passage times based on this remark, as follows. First introduce the class of processes $X$ which are uniquely characterised by a pair $(\mathcal{Q}, g)$, where $\mathcal{Q}$ is a $\sigma$-finite measure and $g$ is the last zero of $X$. We shall then see how one recovers with this approach at once (1.1) and (1.3) and other formulae that appear in [39]. We also give a few extra formulae that can be interesting for applications; additionally we fix a few small inaccuracies which 
appear in the literature by providing the correct assumptions on the underlying filtered probability space. This approach seems to be the most natural one to understand the role of last passage times.

Our paper is organised as follows. In Sect. 2, we introduce a natural class of stochastic processes (which may have jumps), called class $\Sigma$, to study last passage times. We show that these processes are uniquely characterised by a pair $(\mathcal{Q}, g)$, where $\mathcal{Q}$ is a measure and $g$ is the last zero of the process. This result, which solves a conjecture of Madan, Roynette and Yor [21], and which unifies the framework of the generalised Black-Scholes formulae and some problems of penalisation of the Wiener measure by Najnudel, Roynette and Yor [27], was obtained by Najnudel and Nikeghbali in [25]. We also state and prove a special case of this result which was obtained by Cheridito, Nikeghbali and Platen in [7]. The result is very useful in applications, and strong enough to derive almost all our formulae (except those involving the European call option).

In Sect. 3, we show how to recover naturally from our approach the multiplicative characterisation of last passage times obtained by Nikeghbali and Yor in [32] which underlies some of the important results by Madan, Roynette and Yor or presented in the monograph [39]. This derivation is not so surprising but it is new. In particular, we shall see that any end of a predictable set that avoids stopping times can be written as the last time when a nonnegative local martingale having a continuous supremum process, starting at one and vanishing at infinity reaches its maximum. Moreover there exist formulae for the conditional distribution of this random time that looks into the future and for the conditional distribution of the global maximum of the local martingale over the whole trajectory. Very remarkably, these formulae are universal and do not depend on any Markov assumption. We believe that these results are of their own interest. Indeed nonnegative local martingales with no positive jumps (hence having a continuous supremum process) which vanish at infinity occur in different situations in financial modelling; they often model stock prices under a risk-neutral probability measure or they can model benchmarked derivative prices (where the growth-optimal portfolio is used as a numeraire; see [38] for more details and references). Consequently, any information (such as the conditional distribution) on the time when such a process is at its highest or on the value of the global maximum can be valuable.

In Sect. 4, following Profeta, Roynette and Yor [39], we give some examples of explicit computations for the distribution of last passage times of the form $g_{K}$ in (1.2). We also give in this section some new formulae for the distribution of last passage times, together with some examples, based on the multiplicative approach of Nikeghbali and Yor in [32].

\section{Last passage times and processes of the class $\Sigma$}

\subsection{A remarkable measure}

The class of stochastic processes relevant for the current paper consists of submartingales called of class $\Sigma$; these were first introduced by Yor in [43] and some of their main properties were further studied by Nikeghbali in [28]. Let us recall its definition. 
Definition $2.1([28,43])$ Let $\left(\Omega, \mathcal{F},\left(\mathcal{F}_{t}\right)_{t \geq 0}, \mathbb{P}\right)$ be a filtered probability space. A nonnegative submartingale (resp. local submartingale) $X$ is of class $\Sigma$ if it can be decomposed as $X=N+A$, where $N$ and $A$ are $\left(\mathcal{F}_{t}\right)_{t \geq 0}$-adapted processes satisfying the following assumptions:

- $N=\left(N_{t}\right)_{t \geq 0}$ is a càdlàg martingale (resp. local martingale);

- $A=\left(A_{t}\right)_{t \geq 0}$ is a continuous increasing process with $A_{0}=0$;

- The measure $\left(\mathrm{d} A_{t}\right)$ is carried by the set $\left\{t \geq 0: X_{t}=0\right\}$.

We say that $X$ is of class $\Sigma D$ if $X$ is of class $\Sigma$ and of class $(D)$.

One notes that a process of class $\Sigma$ is "almost" a martingale: outside the zeros of $X$, the process $A$ does not increase. In fact, many processes one often encounters fall into this class, e.g. $X_{t}=\left|M_{t}\right|$, where $M$ is a continuous martingale; $X_{t}=\left(M_{t}-K\right)^{+}$when $M$ is a càdlàg local martingale with only positive jumps and $K \in \mathbb{R}$ is a constant; $X_{t}=\bar{M}_{t}-M_{t}$ where $M$ is a local martingale with only negative jumps. Other remarkable families of examples consist of a large class of recurrent diffusions on natural scale (such as some powers of Bessel processes of dimension $\delta \in(0,2)$; see [25]) or of a function of a symmetric Lévy process (in these cases, $A$ is the local time of the diffusion process or Lévy process), or the age process of Brownian motion $W$ in the filtration of the zeros of $W$, namely $\sqrt{t-g_{t}}$, where $g_{t}=\sup \left\{u \leq t: W_{u}=0\right\}$ (for more examples see [28]).

Before giving our characterisation results, we state a simple but useful lemma.

Lemma 2.2 ([28]) Let $X$ be a process of class $\Sigma$ and $f$ a locally bounded nonnegative Borel function. Define $F(x)=\int_{0}^{x} f(y) \mathrm{d} y$. Then $f(A) X$ is again of class $\Sigma$ and decomposes as

$$
f\left(A_{t}\right) X_{t}=\int_{0}^{t} f\left(A_{u}\right) \mathrm{d} N_{u}+F\left(A_{t}\right) .
$$

Proof If $f$ is $\mathcal{C}^{1}$, then an integration by parts yields

$$
\begin{aligned}
f\left(A_{t}\right) X_{t} & =\int_{0}^{t} f\left(A_{u}\right) \mathrm{d} X_{u}+\int_{0}^{t} f^{\prime}\left(A_{u}\right) X_{u} \mathrm{~d} A_{u} \\
& =\int_{0}^{t} f\left(A_{u}\right) \mathrm{d} N_{u}+\int_{0}^{t} f\left(A_{u}\right) \mathrm{d} A_{u}+\int_{0}^{t} f^{\prime}\left(A_{u}\right) X_{u} \mathrm{~d} A_{u} .
\end{aligned}
$$

Since $\left(\mathrm{d} A_{t}\right)$ is carried by the set $\left\{t: X_{t}=0\right\}$, we have $\int_{0}^{t} f^{\prime}\left(A_{u}\right) X_{u} \mathrm{~d} A_{u}=0$. Because we also have $\int_{0}^{t} f\left(A_{u}\right) \mathrm{d} A_{u}=F\left(A_{t}\right)$, we have thus obtained

$$
F\left(A_{t}\right)-f\left(A_{t}\right) X_{t}=-\int_{0}^{t} f\left(A_{u}\right) \mathrm{d} N_{u} .
$$

The general case follows from a density and monotone class argument.

We now show that any process of class $\Sigma$ is uniquely characterised by its last zero and a remarkable measure. We start with an elementary case that we shall briefly 
prove in this survey and which is very useful in applications. This result is in fact an extension of a result by Azéma and Yor [3] and Azéma, Meyer and Yor [2]. (Indeed, in the case when $X$ is of class $\Sigma D$, one could deduce it from part 1 of Theorem 8.1 in [2].)

Theorem 2.3 ([7]) Assume that $\left(\Omega, \mathcal{F},\left(\mathcal{F}_{t}\right)_{t \geq 0}, \mathbb{P}\right)$ satisfies the usual assumptions or the natural conditions. Let $X$ be a process of class $\Sigma$ such that $\lim _{t \rightarrow \infty} X_{t}=X_{\infty}$ exists a.s. and is finite (in particular $N_{\infty}$ and $A_{\infty}$ exist and are a.s. finite). Let

$$
g:=\sup \left\{t: X_{t}=0\right\} \quad \text { with the convention } \sup \emptyset=0 .
$$

1. If $X$ is of class $(D)$, then

$$
X_{T}=\mathbb{E}\left[X_{\infty} \mathbb{1}_{\{g \leq T\}} \mid \mathcal{F}_{T}\right] \text { for every stopping time } T .
$$

2. More generally, if there exists a strictly positive Borel function $f$ such that $\left(f\left(A_{t}\right) X_{t}\right)_{t \geq 0}$ is of class $(D)$, then (2.2) holds.

3. If $\left(N_{t}^{+}\right)_{t \geq 0}$ is of class $(D)$, then (2.2) holds.

Proof (1) For a given stopping time $T$, denote

$$
d_{T}=\inf \left\{t>T: X_{t}=0\right\} \quad \text { with the convention } \inf \emptyset=\infty \text {. }
$$

$d_{T}$ is a stopping time. Since $X_{\infty} \mathbb{1}_{\{g \leq T\}}=X_{d_{T}}$ and $A_{T}=A_{d_{T}}$, it follows from Doob's optional stopping theorem that (recall that since $X$ is of class $(D)$, its martingale part is a uniformly integrable martingale and its increasing process is integrable)

$$
\mathbb{E}\left[X_{\infty} \mathbb{1}_{\{g \leq T\}} \mid \mathcal{F}_{T}\right]=\mathbb{E}\left[N_{d_{T}}+A_{d_{T}} \mid \mathcal{F}_{T}\right]=\mathbb{E}\left[N_{d_{T}}+A_{T} \mid \mathcal{F}_{T}\right]=N_{T}+A_{T}=X_{T}
$$

(2) Assume that there exists a strictly positive Borel function such that $\left(f\left(A_{t}\right) X_{t}\right)_{t \geq 0}$ is of class $(D)$. This property is preserved if one replaces $f$ by a smaller strictly positive Borel function; hence one can suppose that $f$ is locally bounded. Then $\left(f\left(A_{t}\right) X_{t}\right)_{t \geq 0}$ is of class $\Sigma D$, and from part (1) of the theorem, we have

$$
f\left(A_{T}\right) X_{T}=\mathbb{E}\left[f\left(A_{\infty}\right) X_{\infty} \mathbb{1}_{\{g \leq T\}} \mid \mathcal{F}_{T}\right] .
$$

But on the set $\{g \leq T\}$, we have $A_{\infty}=A_{T}$, and consequently

$$
f\left(A_{T}\right) X_{T}=f\left(A_{T}\right) \mathbb{E}\left[X_{\infty} \mathbb{1}_{\{g \leq T\}} \mid \mathcal{F}_{T}\right] .
$$

The result follows by dividing both sides by $f\left(A_{T}\right)$ which is strictly positive.

(3) Since $X \geq 0$ and since $\left(N_{t}^{+}\right)_{t \geq 0}$ is of class $(D)$, we note that $\left(\exp \left(-A_{t}\right) X_{t}\right)_{t \geq 0}$ is of class $(D)$ and the result follows from part (2).

Remark 2.4 With the notation of the introduction, the measure $\mathcal{Q}$ is given by $\mathcal{Q}=X_{\infty} \cdot \mathbb{P}$. 
An interesting case is constructed as follows. Let $M \in \mathcal{M}_{0}$. Then $X=\bar{M}-M$ is of class $\Sigma$ but not of class $(D)$. However, with the notation of Theorem 2.3, $N=-M$ satisfies $N_{t}^{+}=0$ and hence $\mathcal{Q}=\bar{M}_{\infty} . \mathbb{P}$. In this example, the process $X$ is called the drawdown process and is extensively studied in [7]; the interested reader can find there more results related to drawdown and relative drawdown processes and applications to finance.

We now see how the results of [21] and [39] on the prices of put options follow from Theorem 2.3.

Corollary 2.5 (Madan, Roynette and Yor [21]) Let $K$ be a constant and $M a$ local martingale with no positive jumps such that $\left(M_{t}^{-}\right)$is of class $(D)$. Set $g_{K}=\sup \left\{t \geq 0: M_{t}=K\right\}$. Then

$$
\left(K-M_{T}\right)^{+}=\mathbb{E}\left[\left(K-M_{\infty}\right)^{+} \mathbb{1}_{\left\{g_{K} \leq T\right\}} \mid \mathcal{F}_{T}\right]
$$

for every stopping time $T$. In particular, if $M_{\infty}=0$, then

$$
\left(K-M_{T}\right)^{+}=K \mathbb{P}\left[g_{K} \leq T \mid \mathcal{F}_{T}\right] .
$$

Proof $K-M$ is a local martingale with no negative jumps. It follows that $(K-M)^{+}$ is a local submartingale of class $\Sigma$ (see for instance [7]). Since $M^{-}$is of class $\Sigma$, $(K-M)^{+}$is of class $\Sigma D$ and the result follows from Theorem 2.3 by noting that $g_{K}=\sup \left\{t:\left(K-M_{t}\right)^{+}=0\right\}$.

The following extension of Corollary 2.5 has been obtained by Profeta, Roynette and Yor [39] with methods from the theory of enlargement of filtrations. We can deduce it under slightly weaker assumptions from Theorem 2.3.

Corollary 2.6 (Profeta, Roynette and Yor [39]) Let $K^{1}, \ldots, K^{n}$ be positive constants and $M^{1}, \ldots, M^{n}$ nonnegative local martingales that have no positive jumps. Assume that $\left[M^{i}, M^{j}\right]=0$ for $i \neq j$ and denote $g^{i}=\sup \left\{t: M_{t}^{i}=K^{i}\right\}$. Then

$$
\prod_{i=1}^{n}\left(K^{i}-M_{T}^{i}\right)^{+}=\mathbb{E}\left[\prod_{i=1}^{n}\left(K^{i}-M_{\infty}^{i}\right)^{+} \mathbb{1}_{\left\{g^{i} \leq T\right\}} \mid \mathcal{F}_{T}\right]
$$

for every stopping time $T$. In particular, if $M_{\infty}^{i}=0$ for all $i=1, \ldots, n$, then

$$
\prod_{i=1}^{n}\left(K^{i}-M_{T}^{i}\right)^{+}=\prod_{i=1}^{n} K^{i} \mathbb{P}\left[\bigvee_{i=1}^{n} g^{i} \leq T \mid F_{T}\right] .
$$

Proof $X^{i}=\left(K^{i}-M^{i}\right)^{+}$are local submartingales of class $\Sigma$ such that $\left[X^{i}, X^{j}\right]=0$ for $i \neq j$. A simple induction shows that $\prod_{i=1}^{n} X^{i}$ is again of class $\Sigma$ and is bounded. Now (2.3) is a consequence of Theorem 2.3.

We now state a more general and subtle result from [25] linking a process $X$ of class $\Sigma$ and a pair $(\mathcal{Q}, g)$ consisting of the last zero of $X$ and a remarkable $\sigma$-finite 
measure $\mathcal{Q}$. This theorem unifies the results of Madan, Profeta, Roynette and Yor on prices of European put and call options and the results of Najnudel, Roynette and Yor on penalisations of the Wiener measure. It seems the right framework to study call options in terms of last passage times. This new framework is also more general since it contains processes with jumps. For stating this general case, we need to be very careful; the theorem below would be wrong under the usual conditions and it would also be wrong if the filtration $\left(\mathcal{F}_{t}\right)$ does not allow the extension of consistent probability measures. The natural conditions are here used to ensure, in particular, that there exists a continuous and adapted version of the process $A$ which is defined everywhere (think of $A$ being for example the local time at the level 0 of the Wiener process). According to Sect. 1, typical probability spaces where the following theorem holds are $\mathcal{C}\left(\mathbb{R}_{+}, \mathbb{R}\right)$ and $\mathcal{D}\left(\mathbb{R}_{+}, \mathbb{R}\right)$ endowed with the filtration generated by the coordinate process.

Theorem 2.7 (Najnudel and Nikeghbali [25]) Let $X$ be a true submartingale of class $\Sigma$; so its local martingale part $N$ is a true martingale, and $X_{t}$ is integrable for all $t \geq 0$. We suppose that $X$ is defined on a filtered probability space $\left(\Omega, \mathcal{F},\left(\mathcal{F}_{t}\right)_{t \geq 0}, \mathbb{P}\right)$ satisfying the property $(N P)$ (see the Appendix); in particular, this space satisfies the natural conditions and $\mathcal{F}$ is the $\sigma$-algebra generated by $\mathcal{F}_{t}$ for $t \geq 0$. Then there exists a unique $\sigma$-finite measure $\mathcal{Q}$, defined on $(\Omega, \mathcal{F}, \mathbb{P})$, such that for $g:=\sup \left\{t \geq 0: X_{t}=0\right\}$, we have

- $\mathcal{Q}[g=\infty]=0$;

- for all $t \geq 0$, and for all $\mathcal{F}_{t}$-measurable, bounded random variables $\Gamma_{t}$,

$$
\mathcal{Q}\left[\Gamma_{t} \mathbb{1}_{\{g \leq t\}}\right]=\mathbb{E}\left[\Gamma_{t} X_{t}\right] .
$$

Remark 2.8 Theorem 2.7 applies for example when $X$ is the absolute value of Brownian motion, or a Bessel process of dimension $d \in(0,2)$. Indeed the Brownian case follows from Tanaka's formula and the properties of local time, while the lowdimensional Bessel case is deduced from the case of the absolute value of Brownian motion with time change arguments (see e.g. [29] where this is derived carefully). It also applies when $X=|Y|^{\alpha-1}$, where $Y$ is a symmetric stable Lévy process of index $\alpha \in(1,2)$ (this follows from results of Salminen, Yor and Bertoin; see [26] where this is proved and exploited in relation with Theorem 2.7).

In [25], the measure $\mathcal{Q}$ is explicitly constructed in the following way (with a slightly different notation). Let $f$ be a Borel, Lebesgue-integrable, strictly positive and bounded function from $\mathbb{R}$ to $\mathbb{R}$, and define the function $G$ by

$$
G(x)=\int_{x}^{\infty} f(y) \mathrm{d} y .
$$

One can prove that the process

$$
M_{t}^{f}:=G\left(A_{t}\right)-\mathbb{E}\left[G\left(A_{\infty}\right) \mid \mathcal{F}_{t}\right]+f\left(A_{t}\right) X_{t}, \quad t \geq 0,
$$

is a martingale with respect to $\mathbb{P}$ and the filtration $\left(\mathcal{F}_{t}\right)_{t \geq 0}$. Since $\left(\Omega, \mathcal{F},\left(\mathcal{F}_{t}\right)_{t \geq 0}, \mathbb{P}\right)$ satisfies the natural conditions and since $G\left(A_{t}\right) \geq G\left(A_{\infty}\right)$, one can suppose that 
this martingale is nonnegative and càdlàg, by choosing carefully the version of $\mathbb{E}\left[G\left(A_{\infty}\right) \mid \mathcal{F}_{t}\right]$. In this case, since $\left(\Omega, \mathcal{F},\left(\mathcal{F}_{t}\right)_{t \geq 0}, \mathbb{P}\right)$ satisfies the property (NP), there exists a unique finite measure $\mathcal{M}^{f}$ such that for all $t \geq 0$ and for all bounded, $\mathcal{F}_{t}$-measurable functionals $\Gamma_{t}$, we have

$$
\mathcal{M}^{f}\left[\Gamma_{t}\right]=\mathbb{E}\left[\Gamma_{t} M_{t}^{f}\right] .
$$

Now, since $f$ is strictly positive, one can define a $\sigma$-finite measure $\mathcal{Q}^{f}$ by

$$
\mathcal{Q}^{f}:=\frac{1}{f\left(A_{\infty}\right)} \cdot \mathcal{M}^{f} .
$$

It is proved in [25] that if the function $G / f$ is uniformly bounded (this condition is for example satisfied for $f(x)=e^{-x}$ ), then $\mathcal{Q}^{f}$ satisfies the conditions defining $\mathcal{Q}$ in Theorem 2.7, which implies the existence part of that result. The uniqueness part is then proved in a straightforward way; one remarkable consequence of it is the fact that $\mathcal{Q}^{f}$ does not depend on the particular choice of $f$.

Remark 2.9 If $\mathcal{Q}$ is a probability measure, then (2.4) can be written as

$$
X_{t}=\mathbb{E}_{\mathcal{Q}}\left[g \leq t \mid \mathcal{F}_{t}\right] .
$$

Now we are able to state a rigorous and more general version for the price of a European call option when $M$ is a true martingale.

Theorem 2.10 Let $M$ be a (true) nonnegative and continuous martingale on a filtered probability space $\left(\Omega, \mathcal{F},\left(\mathcal{F}_{t}\right)_{t>0}, \mathbb{P}\right)$ satisfying the property $(N P)$. Assume that $M_{0}=1$. Then there exists a probability measure $\mathbb{P}^{(M)}$ such that for all $K>0$ and for all $\mathcal{F}_{t}$-measurable, bounded (or nonnegative) random variables $\Gamma_{t}$, we have

$$
\mathbb{E}\left[\Gamma_{t}\left(M_{t}-K\right)^{+}\right]=\gamma \mathbb{E}^{(M)}\left[\Gamma_{t} \mathbb{1}_{\left\{g_{K} \leq t\right\}}\right],
$$

where

$$
\gamma=1-K+\mathbb{E}\left[\left(K-M_{\infty}\right)^{+}\right] \quad \text { and } \quad g_{K}=\sup \left\{t \geq 0: M_{t}=K\right\} .
$$

In particular,

$$
\mathbb{E}\left[\left(M_{t}-K\right)^{+}\right]=\gamma \mathbb{P}^{(M)}\left[g_{K} \leq t\right] .
$$

The probability measure $\mathbb{P}^{(M)}$ is given by

$$
\mathbb{P}_{\mid \mathcal{F}_{t}}^{(M)}=M_{t} \cdot \mathbb{P}_{\mid \mathcal{F}_{t}}
$$

When $\lim _{t \rightarrow \infty} M_{t}=0$, then $\gamma=1$ and

$$
\mathbb{E}\left[\left(M_{t}-K\right)^{+}\right]=\mathbb{P}^{(M)}\left[g_{K} \leq t\right],
$$

which is the formula obtained by Profeta, Roynette and Yor [39]. Moreover, in this case, we have the remarkable identity

$$
\mathbb{P}^{(M)}\left[g_{K}>t\right]=K \mathbb{P}\left[g_{K}>t\right] .
$$


Proof Because $M$ is a true martingale, $X=(M-K)^{+}$is a true submartingale and therefore Theorem 2.7 applies and gives the existence of a $\sigma$-finite measure $\mathcal{Q}$ such that for $g:=\sup \left\{t \geq 0: X_{t}=0\right\}=g_{K}$, we have

- $\mathcal{Q}\left[g_{K}=\infty\right]=0$;

- for all $t \geq 0$, and for all $\mathcal{F}_{t}$-measurable, bounded random variables $\Gamma_{t}$,

$$
\mathcal{Q}\left[\Gamma_{t} \mathbb{1}_{\left\{g_{K} \leq t\right\}}\right]=\mathbb{E}\left[\Gamma_{t}\left(M_{t}-K\right)^{+}\right] .
$$

Now we note that since $\left(M_{t}-K\right)^{+}=M_{t}-K+\left(K-M_{t}\right)^{+}$, we have

$$
\mathbb{E}\left[\left(M_{t}-K\right)^{+}\right]=1-K+\mathbb{E}\left[\left(K-M_{t}\right)^{+}\right] .
$$

By dominated convergence, $\lim _{t \rightarrow \infty} \mathbb{E}\left[\left(M_{t}-K\right)^{+}\right]=1-K+\mathbb{E}\left[\left(K-M_{\infty}\right)^{+}\right]$. Call this limit $\gamma$. Now taking $\Gamma_{t} \equiv 1$ in (2.4) and then letting $t \rightarrow \infty$, we get $\gamma=\mathcal{Q}\left[\mathbb{1}_{\left\{g_{K}<\infty\right\}}\right]$. But since $\mathcal{Q}\left[g_{K}=\infty\right]=0$, we find that $\mathcal{Q}$ is a finite measure with total mass $\gamma$. Consequently $\mathbb{P}^{(M)}:=\frac{1}{\gamma} \mathcal{Q}$ is a probability measure that satisfies (2.5). Now it remains to check that $\mathbb{P}_{\mid \mathcal{F}_{t}}^{(M)}=M_{t} . \mathbb{P}_{\mid \mathcal{F}_{t}}$. Since the measure $\mathcal{Q}$ is unique, one can directly check that it satisfies the required properties (see [39]).

Remark 2.11 The case when $M$ is a strict local martingale is much more involved. It was dealt with in a special case by Yen and Yor in [41] and was solved in a very general setup by Kardaras, Kreher and Nikeghbali in [17]. In addition to the last passage time, the formula involves the explosion time of $M$ as well (see [17] for more details).

\subsection{Some examples of Azéma supermartingales and some identities in law}

When one studies random times which are not stopping times, there is a supermartingale that plays a crucial role, namely the Azéma supermartingale. More precisely, if $\rho$ is a measurable nonnegative random variable, its Azéma supermartingale is the càdlàg version of $\mathbb{P}\left[\rho>t \mid \mathcal{F}_{t}\right]$. This supermartingale is the key process in the theory of progressive enlargements of filtrations (see e.g. [16] or the survey [31]). It also plays an important role in the modelling of default times in credit risk models (see e.g. $[13,15])$. Given a random time which is not a stopping time, it is in general not possible to compute its Azéma supermartingale. We now give a corollary of Theorem 2.3 which will allow us to compute explicitly the Azéma supermartingale of many last passage times of interest in applications (this method was first used in [30]).

Corollary 2.12 Let $X$ be a process of class $\Sigma D$ satisfying $X_{\infty}=1$ almost surely. Let $g:=\sup \left\{t: X_{t}=0\right\}$. Then

$$
X_{t}=\mathbb{P}\left[g \leq t \mid \mathcal{F}_{t}\right]
$$

Remark 2.13 The proof is straightforward by taking $X_{\infty}=1$ in Theorem 2.3. In particular, we are able to get rid of the assumption that the filtration $\left(\mathcal{F}_{t}\right)$ should be a Brownian-type filtration with only continuous martingales, which is commonly 
imposed in the literature. Moreover, this result can be viewed as a converse to an important result by Azéma [1] which states that one minus the Azéma supermartingale of any end of a predictable set which avoids stopping times is of class $\Sigma D$ and satisfies $X_{\infty}=1$.

We now show how to use the above corollary to compute some Azéma supermartingales.

Example 2.14 Let $M$ be a continuous martingale such that $\langle M, M\rangle_{\infty}=\infty$, and let

$$
T_{1}=\inf \left\{t \geq 0: M_{t}=1\right\} \quad \text { and } \quad g=\sup \left\{t<T_{1}: M_{t}=0\right\} .
$$

Then $\left(M_{t \wedge T_{1}}^{+}\right)$satisfies the conditions of Corollary 2.12, and hence

$$
\mathbb{P}\left[g \leq t \mid \mathcal{F}_{t}\right]=M_{t \wedge T_{1}}^{+}=\int_{0}^{t \wedge T_{1}} \mathbb{1}_{\left\{M_{u}>0\right\}} \mathrm{d} M_{u}+\frac{1}{2} L_{t \wedge T_{1}},
$$

where $L$ is the local time of $M$ at 0 .

One can also consider

$$
T_{ \pm 1}=\inf \left\{t \geq 0:\left|M_{t}\right|=1\right\} \quad \text { and } \quad \tau=\sup \left\{t<T_{ \pm 1}:\left|M_{t}\right|=0\right\}
$$

Then $\left(\left|M_{t \wedge T_{ \pm 1}}\right|\right)$ satisfies the conditions of Corollary 2.12, hence

$$
\mathbb{P}\left[\tau \leq t \mid \mathcal{F}_{t}\right]=\left|M_{t \wedge T_{ \pm 1}}\right|=\int_{0}^{t \wedge T_{ \pm 1}} \operatorname{sign}\left(M_{u}\right) \mathrm{d} M_{u}+L_{t \wedge T_{ \pm 1}}
$$

Example 2.15 Let $Y$ be a real continuous recurrent diffusion process with $Y_{0}=0$. Then from the general theory of diffusion processes, there exists a unique (continuous and strictly increasing) scale function $s$ with $s(0)=0, \lim _{x \rightarrow+\infty} s(x)=+\infty$, $\lim _{x \rightarrow-\infty} s(x)=-\infty$ and such that $s(Y)$ is a continuous local martingale. Let

$$
T_{1}=\inf \left\{t \geq 0: Y_{t}=1\right\} \text {. }
$$

Now if we define

$$
X_{t}:=\frac{s\left(Y_{t \wedge T_{1}}\right)^{+}}{s(1)},
$$

we easily note that $X$ is a local submartingale of class $\Sigma$ which satisfies the hypotheses of Corollary 2.12. Consequently for

$$
g=\sup \left\{t<T_{1}: Y_{t}=0\right\},
$$

we have

$$
\mathbb{P}\left[g \leq t \mid \mathcal{F}_{t}\right]=\frac{s\left(Y_{t \wedge T_{1}}\right)^{+}}{s(1)}
$$


Example 2.16 Let $M$ be a positive local martingale with $M_{0}=x>0$ and $\lim _{t \rightarrow \infty} M_{t}=0$. Then Tanaka's formula shows that $\left(1-\frac{M}{y} \wedge 1\right)$, for $0 \leq y \leq x$, is a local submartingale of class $\Sigma$ satisfying the assumptions of Corollary 2.12. Hence with

$$
g=\sup \left\{t: M_{t}=y\right\},
$$

we have

$$
\mathbb{P}\left[g>t \mid \mathcal{F}_{t}\right]=\frac{M_{t}}{y} \wedge 1=1+\frac{1}{y} \int_{0}^{t} \mathbb{1}_{\left\{M_{u}<y\right\}} \mathrm{d} M_{u}-\frac{1}{2 y} L_{t}^{y},
$$

where $L^{y}$ is the local time of $M$ at $y$.

Example 2.17 As an illustration of the previous example, consider a transient diffusion $R$ with values in $[0, \infty)$ which has $\{0\}$ as entrance boundary. Let $s$ be a scale function for $R$, which we can choose such that $s(0)=-\infty$, and $s(\infty)=0$. Then, under the law $\mathbb{P}_{x}$, for any $x>0$, the local martingale $M=-s(R)$ satisfies the conditions of the previous example and for $x, y \geq 0$, we have

$$
\mathbb{P}_{x}\left[g_{y}>t \mid \mathcal{F}_{t}\right]=\frac{s\left(R_{t}\right)}{s(y)} \wedge 1=1+\frac{1}{s(y)} \int_{0}^{t} \mathbb{1}_{\left\{R_{u}>y\right\}} \mathrm{d}\left(s\left(R_{u}\right)\right)+\frac{1}{2 s(y)} L_{t}^{s(y)},
$$

where $L^{s(y)}$ is the local time of $s(R)$ at $s(y)$ and $g_{y}=\sup \left\{t: R_{t}=y\right\}$. This last formula was the key point for deriving the distribution of $g_{y}$ in [34], Theorem 6.1.

The structure of the class $\Sigma$ together with the examples above suggests that the increasing process $A$ should have some interesting properties. The examples we have considered so far show that $A$ can be the local time of a martingale, a diffusion process, or a Lévy process. It can also be the supremum process of a local martingale with only negative jumps. Hence it would be interesting to have some information about the distribution of $A_{\infty}$ when it is finite. This is clearly the case for the examples above and more generally for processes of class $\Sigma D$. In many situations, by stopping, one is in the situation where $X_{\infty}=\varphi\left(A_{\infty}\right)$; this occurs in the resolution of the Skorokhod problem by Azéma and Yor (see e.g. [40]), or in the resolution of the Skorokhod problem for processes of class $\Sigma$ (see [28]). It also appears naturally around problems related to the drawdown or relative drawdown processes (see [7]). All these situations are covered by the following theorem.

Theorem 2.18 ([7]) Let $X$ be a process of class $\Sigma$ and assume that there exists a locally bounded Borel function $f: \mathbb{R}_{+} \rightarrow \mathbb{R}_{+}$such that the process $f(A) X$ is of class $\Sigma D$ and $\lim _{t \rightarrow \infty} f\left(A_{t}\right) X_{t}=1$ almost surely. Set $F(x)=\int_{0}^{x} f(y) \mathrm{d} y$ and assume $F(\infty)=\infty$. Then for every stopping time $T$ and for all Borel functions $h:[0, a) \rightarrow \mathbb{R}$ satisfying

$$
\int_{0}^{\infty}|h(y)| e^{-F(y)} \mathrm{d} F(y)<\infty,
$$


one has

$$
\begin{aligned}
\mathbb{E}\left[h\left(A_{\infty}\right) \mid \mathcal{F}_{T}\right]= & h(0) f(0) X_{0}+h^{F}(0)\left(1-f(0) X_{0}\right) \\
& +\int_{0}^{T}\left(h-h^{F}\right)\left(A_{u}\right) f\left(A_{u}\right) \mathrm{d} N_{u} \\
= & h\left(A_{T}\right) f\left(A_{T}\right) X_{T}+h^{F}\left(A_{T}\right)\left(1-f\left(A_{T}\right) X_{T}\right),
\end{aligned}
$$

where

$$
h^{F}(x)=e^{F(x)} \int_{x}^{\infty} h(y) e^{-F(y)} \mathrm{d} F(y), \quad x \geq 0 .
$$

In particular, conditionally on $\mathcal{F}_{T}$, the law of $A_{\infty}$ is given by

$$
\mathbb{P}\left[A_{\infty}>x \mid \mathcal{F}_{T}\right]=\mathbb{1}_{\left\{A_{T}>x\right\}}+\mathbb{1}_{\left\{A_{T} \leq x\right\}}\left(1-f\left(A_{T}\right) X_{T}\right) e^{F\left(A_{T}\right)-F(x)}, \quad x \geq 0 .
$$

We postpone an important application of the above theorem to the next section.

\section{The multiplicative approach}

There also exists a simple multiplicative approach to study ends of predictable sets; it was developed by Nikeghbali and Yor in [32] and underlies some of the techniques used by Madan, Profeta, Roynette and Yor in their studies of the generalised BlackScholes formulae [21, 22, 39]. This multiplicative approach has also been used by Coculescu and Nikeghbali in [9] to obtain general formulae when the default time in credit risk models is modelled by the end of a predictable set. It was also used more recently by Li and Rutkowski in [20]. The idea in this approach is to consider a multiplicative decomposition for the Azéma supermartingale of the end of a predictable set rather than the (additive) Doob-Meyer decomposition. As a consequence, every end of a predictable set has an intuitive representation in terms of some simple and intuitive last passage time. We deduce these results from those of the previous section. This is new and as a consequence we are able to avoid the assumption that the filtration $\left(\mathcal{F}_{t}\right)_{t \geq 0}$ contains only continuous martingales.

Proposition 3.1 Let $M \in \mathcal{M}_{0}$. Consider

$$
g=\sup \left\{t \geq 0: M_{t}=\bar{M}_{t}\right\}=\sup \left\{t \geq 0: M_{t}=\bar{M}_{\infty}\right\} .
$$

Then the Azéma supermartingale of $g$ is given by

$$
Z_{t}:=\mathbb{P}\left[g>t \mid \mathcal{F}_{t}\right]=\frac{M_{t}}{\bar{M}_{t}}=1+\int_{0}^{t} \frac{\mathrm{d} M_{s}}{\bar{M}_{s}}-\log \bar{M}_{t} .
$$

Proof The result follows directly from Theorem 2.3 when applied to $X=1-\frac{M}{\bar{M}}$ which is of class $\Sigma D$.

The remarkable feature of the above family of examples is that it actually covers all cases of ends of optional sets which avoid stopping times: 
Theorem 3.2 Let $g$ be the end of an optional set which avoids stopping times, that is, $\mathbb{P}[g=T>0]=0$ for all $\left(\mathcal{F}_{t}\right)$-stopping times $T$. Then there exists a unique local martingale $M \in \mathcal{M}_{0}$ such that

$$
g=\sup \left\{t \geq 0: M_{t}=\bar{M}_{t}\right\} \quad \text { and } \quad \mathbb{P}\left[g>t \mid \mathcal{F}_{t}\right]=\frac{M_{t}}{\bar{M}_{t}}=Z_{t}
$$

Proof It is a well-known result of Azéma [1] that $1-\mathbb{P}\left[g>t \mid \mathcal{F}_{t}\right]=: X_{t}$ is of class $\Sigma$. Let us write $X=N+A$. Now it follows from (2.1) that

$$
\exp \left(A_{t}\right) X_{t}=\int_{0}^{t} \exp \left(A_{u}\right) \mathrm{d} N_{u}+\exp \left(A_{t}\right)-1
$$

or equivalently

$$
\exp \left(A_{t}\right)\left(1-X_{t}\right)=1-\int_{0}^{t} \exp \left(A_{u}\right) \mathrm{d} N_{u}
$$

Let us write $M=1-\int \exp \left(A_{u}\right) \mathrm{d} N_{u}$. This is a local martingale starting from 1 . Using the above representation $M_{t}=\exp \left(A_{t}\right)\left(1-X_{t}\right)$, we see that $\bar{M}_{t}=\exp \left(A_{t}\right)$ since $A$ is continuous, increasing, and increases only on the zeros of $X$ (hence $\bar{M}$ is continuous). Hence it follows from (3.1) that

$$
Z_{t}=1-X_{t}=\frac{M_{t}}{\bar{M}_{t}}
$$

The above representation also implies that $\lim _{t \rightarrow \infty} M_{t}=0$, which completes the proof.

Remark 3.3 In modelling the default time in credit risk modelling, it is important to know when the hazard process and the martingale hazard process are different. In [9], Theorem 3.2 is used to show that under the assumption that the end of an optional set $g$ avoids stopping times, the martingale hazard process and the hazard process are always different and this difference is explicitly computed (in fact in [9], it is assumed that the filtration $\left(\mathcal{F}_{t}\right)$ is such that all martingales are continuous; our slightly improved version shows that one can avoid this assumption). Theorem 3.2 is also used in [9] to compute the price of defaultable claims.

In [32], Doob's maximal identity (which states that for a local martingale in $\mathcal{M}_{0}$ the distribution of $\bar{M}_{\infty}$ is the same as the inverse of a uniform random variable on $(0,1))$ is used to derive the conditional distribution of $\bar{M}_{\infty}$, which is a crucial step in some problems of enlargements of filtrations. Here we shall deduce this from the more general Theorem 2.18. This result seems to be important enough on its own to be included in this survey. It is also valuable in the context where prices under the risk-neutral probability measure as well as benchmarked portfolios are local martingales in $\mathcal{M}_{0}$, to have information on the conditional distribution of the maximum of martingales in $\mathcal{M}_{0}$. 
Proposition 3.4 ([32]) Let $M \in \mathcal{M}_{0}$. For any Borel bounded or positive function $f$, we have

$$
\begin{aligned}
\mathbb{E}\left[f\left(\bar{M}_{\infty}\right) \mid \mathcal{F}_{t}\right] & =f\left(\bar{M}_{t}\right)\left(1-\frac{M_{t}}{\bar{M}_{t}}\right)+\int_{0}^{M_{t} / \bar{M}_{t}} \mathrm{~d} x f\left(\frac{M_{t}}{x}\right) \\
& =f\left(\bar{M}_{t}\right)\left(1-\frac{M_{t}}{\bar{M}_{t}}\right)+M_{t} \int_{\bar{M}_{t}}^{\infty} \mathrm{d} x \frac{f(x)}{x^{2}} .
\end{aligned}
$$

Moreover we have the representation of $\mathbb{E}\left[f\left(\bar{M}_{\infty}\right) \mid \mathcal{F}_{t}\right]$ as a stochastic integral,

$$
\mathbb{E}\left[f\left(\bar{M}_{\infty}\right) \mid \mathcal{F}_{t}\right]=\mathbb{E}\left[f\left(\bar{M}_{\infty}\right)\right]+\int_{0}^{t} g\left(\bar{M}_{S}\right) \mathrm{d} M_{s},
$$

where $g$ is given by $g(x)=\int_{x}^{\infty} \frac{\mathrm{d} y}{y^{2}}(f(y)-f(x))$.

Proof The proof follows immediately from Theorem 2.18 applied to the process

$$
X_{t}=1-\frac{M_{t}}{\bar{M}_{t}}=-\int_{0}^{t} \frac{\mathrm{d} M_{s}}{\bar{M}_{s}}+\log \bar{M}_{t} .
$$

Indeed in this case we take $h(x)=f(\exp (x))$ in Theorem 2.18, with $N=-\int \frac{\mathrm{d} M_{s}}{\bar{M}_{s}}$ and $A=\log \bar{M}$.

Remark 3.5 By taking $t=0$ above we obtain the well-known identity in law

$$
\bar{M}_{\infty} \stackrel{\text { law }}{=} \frac{1}{U}
$$

where $U$ is a uniform random variable on $(0,1)$.

Remark 3.6 Formulae (3.2) and (3.3) suggest that there should exist ways of creating new financial products with robust prices due to model independence. For instance one could imagine an option with payoff $f\left(\bar{M}_{\infty}\right)$ in areas where modelling risk over a long period has been of much concern, as in pension fund and insurance problems. In this case formulae (3.2) and (3.3) give the price of the option together with a hedging strategy. We emphasise again that these formulae are very robust since they do not depend on the underlying dynamics of the discounted or benchmarked stock price $M$.

\section{Distributions of last passage times}

At this point in our discussion, the following questions can be raised:

- The last passage time $g_{K}$ defined by (1.2) plays a central role in generalised BlackScholes formulae. Can one compute the law of such times for a wide range of examples? 
- Theorem 3.2 gives a simple general representation for the Azéma supermartingale of ends of optional sets that avoid stopping times. Can this be used to obtain a systematic way to compute the law of such a random time?

The next subsections give some answers to the above questions.

4.1 Some examples for distributions of $g_{K}$

In this paragraph, we follow closely the computations in the monograph by Profeta, Roynette and Yor [39, Sect. 2.4] to obtain a family of interesting examples.

We assume that $M$ is in $\mathcal{M}_{0}$ and continuous. We make the following additional assumptions:

(i) For every $t>0$, the law of the random variable $M_{t}$ admits a density $\left(m_{t}(x), x \geq 0\right)$ and $(t, x) \mapsto m_{t}(x)$ may be chosen continuous on $(0, \infty)^{2}$.

(ii) The quadratic covariation $\langle M, M\rangle$ of $M$ satisfies

$$
\mathrm{d}\langle M, M\rangle_{t}=\sigma_{t}^{2} \mathrm{~d} t .
$$

We further assume that there exists a jointly continuous function

$$
(t, x) \mapsto \theta_{t}(x):=\mathbb{E}\left[\sigma_{t}^{2} \mid M_{t}=x\right]
$$

on $(0, \infty)^{2}$.

Theorem 4.1 (Profeta, Roynette and Yor [39]) Under the preceding hypotheses, the law of $g_{K}$ is given by

$$
\mathbb{P}\left[g_{K} \in \mathrm{d} t\right]=\left(1-\frac{a}{K}\right)^{+} \delta_{0}(\mathrm{~d} t)+\frac{1}{2 K} \theta_{t}(K) m_{t}(K) \mathbb{1}_{\{t>0\}} \mathrm{d} t,
$$

where $a=M_{0}$ and $\delta_{0}$ is the Dirac measure at 0 .

Proof Using Tanaka's formula we have

$$
\mathbb{E}\left[\left(K-M_{t}\right)^{+}\right]=(K-a)^{+}+\frac{1}{2} \mathbb{E}\left[L_{t}^{K}\right],
$$

where $\left(L_{t}^{K}, t \geq 0, K \geq 0\right)$ denotes the in $t$ continuous family of local times of the martingale $M$. Thus it follows from (1.1) that

$$
\mathbb{P}\left[g_{K} \in \mathrm{d} t\right]=\left(1-\frac{a}{K}\right)^{+} \delta_{0}(\mathrm{~d} t)+\frac{\mathbb{1}_{\{t>0\}}}{2 K} \mathrm{~d}_{t} \mathbb{E}\left[L_{t}^{K}\right],
$$

and it remains to show that

$$
\mathrm{d}_{t} \mathbb{E}\left[L_{t}^{K}\right]=\theta_{t}(K) m_{t}(K) \mathrm{d} t .
$$

To prove the above formula we note that for every Borel $f: \mathbb{R}_{+} \rightarrow \mathbb{R}_{+}$, the occupation density formula yields

$$
\int_{0}^{t} f\left(M_{s}\right) \mathrm{d}\langle M, M\rangle_{s}=\int_{0}^{\infty} f(K) L_{t}^{K} \mathrm{~d} K
$$


Under the assumption (ii), the above equality becomes

$$
\int_{0}^{t} f\left(M_{s}\right) \sigma_{s}^{2} \mathrm{~d} s=\int_{0}^{\infty} f(K) L_{t}^{K} \mathrm{~d} K
$$

Taking expectation on both sides we obtain

$$
\mathbb{E}\left[\int_{0}^{t} f\left(M_{s}\right) \sigma_{s}^{2} \mathrm{~d} s\right]=\int_{0}^{\infty} f(K) \mathbb{E}\left[L_{t}^{K}\right] \mathrm{d} K .
$$

But under our assumptions, we can also write

$$
\begin{aligned}
\mathbb{E}\left[\int_{0}^{t} f\left(M_{s}\right) \sigma_{s}^{2} \mathrm{~d} s\right] & =\int_{0}^{t} \mathbb{E}\left[f\left(M_{s}\right) \mathbb{E}\left[\sigma_{s}^{2} \mid M_{s}\right]\right] \mathrm{d} s \\
& =\int_{0}^{\infty} f(K) \mathrm{d} K \int_{0}^{t} \theta_{s}(K) m_{s}(K) \mathrm{d} s .
\end{aligned}
$$

Since our formulae hold for every Borel $f: \mathbb{R}_{+} \rightarrow \mathbb{R}_{+}$, we can thus conclude that

$$
\mathbb{E}\left[L_{t}^{K}\right]=\int_{0}^{t} \theta_{s}(K) m_{s}(K) \mathrm{d} s
$$

which proves the theorem.

We now give some examples.

Example 4.2 Consider $M_{t}=\exp \left(W_{t}-t / 2\right)$, where $W$ is Brownian motion. From Itô's formula, we have $M=1+\int M_{s} \mathrm{~d} W_{s}$, thus $\mathrm{d}\langle M, M\rangle_{t}=M_{t}^{2} \mathrm{~d} t$, and we may apply Theorem 4.1 with $\theta_{t}(x)=x^{2}$ and

$$
m_{t}(x)=\frac{1}{x \sqrt{2 \pi t}} \exp \left(-\frac{1}{2 t}\left(\log x+\frac{t}{2}\right)^{2}\right) .
$$

We thus obtain

$$
\mathbb{P}\left[g_{K} \in \mathrm{d} t\right]=\left(1-\frac{1}{K}\right)^{+} \delta_{0}(\mathrm{~d} t)+\frac{\mathbb{1}_{\{t>0\}}}{2 \sqrt{2 \pi t}} \exp \left(-\frac{1}{2 t}\left(\log K+\frac{t}{2}\right)^{2}\right) \mathrm{d} t .
$$

Example 4.3 We come back to the case of transient diffusions considered in Example 2.17 (recall that $s$ is the scale function). We assume further that the infinitesimal generator of our diffusion has the form

$$
\Gamma=\frac{1}{2} a(x) \frac{\partial^{2}}{\partial x^{2}}+b(x) \frac{\partial}{\partial x} .
$$

In this case, if we denote by $p(t, x, y)$ the density of the random variable $X_{t}$ with respect to Lebesgue measure $\mathrm{d} y$, then the distribution of $g_{K}$ under $\mathbb{P}_{x}$ is (see [39, Sect. 2.5])

$$
\mathbb{P}_{x}\left[g_{K} \in \mathrm{d} t\right]=\left(1-\frac{s(x)}{s(K)}\right)^{+} \delta_{0}(\mathrm{~d} t)-\frac{s^{\prime}(K) a(K)}{2 s(K)} p(t, x, K) \mathrm{d} t .
$$


For instance if $M$ is a Bessel process, i.e., $a=1$ and $b(x)=\frac{2 v+1}{2 x}$, with index $v>0$ (i.e., with dimension $d=2 v+2>2$ ), we have $s(x)=-x^{-2 v}$ and

$$
p(t, 0, K)=\frac{2^{-v}}{\Gamma(v+1)} t^{-(v+1)} K^{2 v+1} \exp \left(-\frac{-K^{2}}{2 t}\right) .
$$

Hence

$$
\begin{aligned}
\mathbb{P}_{0}\left[g_{K} \in \mathrm{d} t\right] & =\frac{v 2^{-v}}{\Gamma(v+1)} \frac{1}{K} \frac{K^{2 v+1}}{t^{v+1}} \exp \left(-\frac{-K^{2}}{2 t}\right) \mathrm{d} t \\
& =\frac{2^{-v}}{\Gamma(v)} \frac{K^{2 v}}{t^{v+1}} \exp \left(-\frac{-K^{2}}{2 t}\right) \mathrm{d} t .
\end{aligned}
$$

A few more examples based on Theorem 4.1 are computed in [39].

4.2 The general distribution of last passage times

We now use Theorem 3.2 to obtain a representation for the law of an arbitrary end of an optional set which avoids stopping times. Indeed, we know from Theorem 3.2 that any such time is the last time when a local martingale in $\mathcal{M}_{0}$ is equal to its running maximum. We start with a simple lemma.

Lemma 4.4 Under the assumptions of Theorem 3.2, the law of the last passage time $g$ is given by

$$
\mathbb{P}[g \leq t]=\mathbb{E}\left[\log \bar{M}_{t}\right]
$$

Proof This follows immediately by taking the expectation of $Z_{t}$ in Theorem 3.2.

We now state and prove a theorem that can be very useful in practice to compute the law of a last passage time.

Theorem 4.5 Under the assumptions of Theorem 3.2 define $\tau_{a}=\inf \left\{t: M_{t}>a\right\}$ for $a \geq 1$. Then for any bounded or positive Borel function $f$, we have

$$
\mathbb{E}[f(g)]=\int_{1}^{\infty} \mathbb{E}\left[f\left(\tau_{a}\right) \mathbb{1}_{\left\{\tau_{a}<\infty\right\}}\right] \frac{\mathrm{d} a}{a} .
$$

In particular, the Laplace transform of the law of $g$ is given by

$$
\mathbb{E}[\exp (-\lambda g)]=\int_{1}^{\infty} \mathbb{E}\left[\exp \left(-\lambda \tau_{a}\right)\right] \frac{\mathrm{d} a}{a} \quad \text { for } \lambda>0 .
$$

Proof We differentiate (4.1) to obtain

$\mathbb{E}[f(g)]=\mathbb{E}\left[\int_{0}^{\infty} f(s) \frac{\mathrm{d} \bar{M}_{s}}{\bar{M}_{s}}\right]=\mathbb{E}\left[\int_{1}^{\bar{M}_{\infty}} f\left(\tau_{a}\right) \frac{\mathrm{d} a}{a}\right]=\int_{1}^{\infty} \mathbb{E}\left[f\left(\tau_{a}\right) \mathbb{1}_{\left\{\tau_{a}<\infty\right\}} \frac{\mathrm{d} a}{a}\right.$. 
Theorem 4.5 allows us to derive some explicit examples for the law of a last passage time when the underlying nonnegative local martingale $M$ belongs to some class of well-known diffusions. We begin with the standard asset price model in finance, the Black-Scholes model. We set

$$
M_{t}=\exp \left(2 \sigma W_{t}-2 \sigma^{2} t\right),
$$

which follows a geometric Brownian motion for $t \geq 0$. The last passage time considered here is the time of the total maximum of $M$, given as

$$
g=\sup \left\{t \geq 0: W_{t}-\sigma t=\sup _{s \geq 0}\left(W_{s}-\sigma s\right)\right\} .
$$

Proposition 4.6 The law of $g$ is characterised by its Laplace transform

$$
\mathbb{E}[\exp (-\lambda g)]=\frac{2}{1+\sqrt{1+\frac{2 \lambda}{\sigma^{2}}}} \text { for } \lambda \geq 0 \text {. }
$$

Proof We use (4.2). The Laplace transform of $\tau_{a}=\inf \left\{t: M_{t}>a\right\}$ is given for instance in [6] as

$$
\mathbb{E}\left[\exp \left(-\lambda \tau_{a}\right)\right]=\left(\frac{1}{a}\right)^{\frac{1}{2}+\sqrt{\frac{1}{4}+\frac{\lambda}{2 \sigma^{2}}}}
$$

for $a>1$ and $\lambda \geq 0$. Substituting (4.4) into (4.2) yields

$$
\begin{aligned}
\mathbb{E}[\exp (-\lambda g)] & =\int_{1}^{\infty}\left(\frac{1}{a}\right)^{\frac{1}{2}+\sqrt{\frac{1}{4}+\frac{\lambda}{2 \sigma^{2}}}} \frac{\mathrm{d} a}{a} \\
& =\int_{0}^{\infty} e^{-u+\left(\frac{1}{2}+\sqrt{\frac{1}{4}+\frac{\lambda}{2 \sigma^{2}}}\right)} \mathrm{d} u=\frac{2}{1+\sqrt{1+\frac{2 \lambda}{\sigma^{2}}}} .
\end{aligned}
$$

Remark 4.7 It is interesting to note that according to (4.3), the time $g$ has the same law as the first hitting time by a Brownian motion with drift of a level twice the value of an independent exponential random variable $\tilde{e}$, i.e.,

$$
g \stackrel{\text { law }}{=} \frac{1}{\sigma^{2}} T_{2 \tilde{e}}
$$

with $T_{a}=\inf \left\{t: \tilde{W}_{t}+t=a\right\}$, where $\tilde{W}$ follows a standard Brownian motion.

One can sometimes reduce the problem of finding the law of the end of a predictable set to that of a geometric Brownian motion after a time change.

Proposition 4.8 Assume that the hypotheses of Theorem 3.2 hold and further that all martingales of the filtration $\left(\mathcal{F}_{t}\right)_{t \geq 0}$ are continuous. Then there exists a unique 
local martingale $D$ with $\langle D\rangle_{\infty}=\infty$ a.s. and $D_{t}=\int_{0}^{t} \frac{\mathrm{d} M_{u}}{M_{u}}=W_{\langle D\rangle_{t}}$, where $W$ is an $\left(\mathcal{F}_{\langle D\rangle_{u}^{-1}}\right)$-Brownian motion, such that

$$
g=\sup \left\{t: W_{\langle D\rangle_{t}}-\frac{1}{2}\langle D\rangle_{t}=\sup _{s \geq 0}\left(W_{\langle D\rangle_{s}}-\frac{1}{2}\langle D\rangle_{s}\right)\right\} .
$$

Proof It is a standard fact of stochastic calculus that there exists a local martingale $D$ such that $\langle D\rangle_{\infty}=\infty$ and $M=\exp \left\{D-\frac{1}{2}\langle D\rangle\right\}$. Moreover, the local martingale $D$ is unique and $D=\int \frac{\mathrm{d} M_{u}}{M_{u}}$. From the Dambis-Dubins-Schwarz theorem, there exists then an $\left(\mathcal{F}_{\langle D\rangle_{u}^{-1}}\right)$-Brownian motion $W$ in $\langle D\rangle$-time such that $D_{t}=W_{\langle D\rangle_{t}}$. If we denote by $\langle D\rangle_{u}^{-1}$ the generalised inverse of $\langle D\rangle_{t}$ defined by

$$
\langle D\rangle_{u}^{-1}=\inf \left\{t \geq 0:\langle D\rangle_{t}>u\right\},
$$

then we can define the last passage time

$$
L=\sup \left\{t \geq 0: W_{t}-\frac{1}{2} t=\sup _{s \geq 0}\left(W_{s}-\frac{1}{2} s\right)\right\} .
$$

Consequently, $g=\langle D\rangle_{L}^{-1}$ is also given by

$$
g=\sup \left\{t: W_{\langle D\rangle_{t}}-\frac{1}{2}\langle D\rangle_{t}=\sup _{s \geq 0}\left(W_{\langle D\rangle_{s}}-\frac{1}{2}\langle D\rangle_{s}\right)\right\} .
$$

Squared Bessel processes play an essential role in various financial models. This includes for instance the constant elasticity of variance model, see [10]; affine models, see [12]; and the minimal market model, see [36, 37]. To study last passage times in some of these models, let $R^{2}$ denote a squared Bessel process of dimension $\delta>2$. In this case $R^{2}$ is transient; see [40, Chap. X]. Furthermore, for any squared Bessel process with index $v=\frac{\delta}{2}-1>0$, the process $M$ with

$$
M_{t}=\left(\frac{R_{0}^{2}}{R_{t}^{2}}\right)^{v}
$$

is a nonnegative, strict local martingale in $\mathcal{M}_{0}$. By using Proposition 3.1, one obtains for $g=\sup \left\{t \geq 0: R_{t}^{2}=I_{t}\right\}$ with $I_{t}=\inf _{s \leq t} R_{s}^{2}$ the conditional probability

$$
\mathbb{P}\left[g>t \mid \mathcal{F}_{t}\right]=\left(\frac{I_{t}}{R_{t}^{2}}\right)^{v},
$$

for all $t \geq 0$. Moreover it follows from Remark 3.5 that the random limit $1 / I_{\infty}$ is uniformly distributed on $(0,1)$ for the case of dimension $\delta=4$. This is an interesting observation for the rather realistic minimal market model, where such dynamics arise.

Proposition 4.9 The Laplace transform of the last passage time $g$ in (4.5) is of the form 


$$
\mathbb{E}[\exp (-\lambda g)]=\frac{2 \nu K_{v}(\sqrt{2 \lambda x})}{(2 \lambda x)^{\frac{v}{2}}} \int_{0}^{\sqrt{2 \lambda x}} \frac{u^{\nu-1}}{K_{v}(u)} \mathrm{d} u \text { for } \lambda>0,
$$

where $R_{0}^{2}=x$ and $K_{v}(\cdot)$ is the modified Bessel function of the second kind.

Proof We first recall from [18] and [6, Part 2, Sect. 6] the Laplace transform of the random variable $\tau_{a}=\inf \left\{t \geq 0: M_{t}=a\right\}=\inf \left\{t \geq 0: R_{t}^{2}=\frac{x}{a^{\frac{1}{v}}}\right\}, a \geq x^{\nu}$, in the form

$$
\mathbb{E}\left[\exp \left(-\lambda \tau_{a}\right)\right]=\frac{K_{v}(\sqrt{2 \lambda x})}{a K_{v}\left(\frac{\sqrt{2 \lambda x}}{a^{\frac{1}{v}}}\right)} \text { for } \lambda>0 .
$$

A combination of (4.7) and (4.2) gives (4.6).

In the special case of dimension $\delta=4$, as it arises for the stylised minimal market model in [36, 37], we have $v=1$ and it follows that

$$
\mathbb{E}[\exp (-\lambda g)]=\frac{2 K_{1}(\sqrt{2 \lambda x})}{\sqrt{2 \lambda x}} \int_{0}^{\sqrt{2 \lambda x}} \frac{1}{K_{1}(u)} \mathrm{d} u .
$$

Another interesting special case is obtained for the squared Bessel process of dimension $\delta=3$, where we are able to provide the following explicit formula for the density.

Corollary 4.10 For dimension $\delta=3$, the law of the last passage time $g$ in (4.5) has the density

$$
p(t)=\frac{1}{\sqrt{2 \pi x t}}\left(1-\exp \left(\frac{-x}{2 t}\right)\right),
$$

where $R_{0}^{2}=x>0$ and $t \geq 0$.

Proof For the squared Bessel process of dimension $\delta=3$, we have $v=\frac{1}{2}$ and from (4.7)

$$
\mathbb{E}_{x}[\exp (-\lambda g)]=\frac{2}{\sqrt{2 \lambda x}} \exp \left(-\frac{\sqrt{2 \lambda x}}{2}\right) \sinh \left(\frac{\sqrt{2 \lambda x}}{2}\right) .
$$

The linearity of the Laplace transform and a close look at a table of inverse Laplace transforms, see for instance [6, Part 2, Sect. 5], then yields (4.8).

Note that the density in (4.8) is dependent on the initial level of the squared Bessel process. This is different from the case of geometric Brownian motion.

We end this section by considering again the general case of a transient diffusion $Y$. Recall that it generates a local martingale $M$ in $\mathcal{M}_{0}$ via the ratio $M_{t}=\frac{s\left(Y_{t}\right)}{s(x)}$, $t \geq 0, Y_{0}=x>0$. Here $s(\cdot)$ is the differentiable scale function of $Y$ which we can choose such that $s(0)=-\infty$ and $s(\infty)=0$. Then we have by Proposition 3.1 again

$$
\mathbb{P}\left[g>t \mid \mathcal{F}_{t}\right]=\frac{s\left(Y_{t}\right)}{s\left(Z_{t}\right)},
$$


where $Z_{t}=\inf _{s \leq t} Y_{s}$ and $g$ is defined as $g=\sup \left\{t \geq 0: Y_{t}=Z_{t}\right\}$. The law of the last passage time $g$ can then be characterised as follows.

Proposition 4.11 The Laplace transform of the above last passage time $g$ is of the form

$$
\mathbb{E}_{x}[\exp (-\lambda g)]=-\int_{0}^{x} \frac{s^{\prime}(u)}{s(u)} \frac{\varphi_{\lambda}(x)}{\varphi_{\lambda}(u)} \mathrm{d} u \text { for } \lambda>0 .
$$

Here $\varphi_{\lambda}(\cdot)$ is a continuous solution of the equation

$$
G \varphi_{\lambda}(y)=\lambda \varphi_{\lambda}(y),
$$

with $G$ denoting the infinitesimal generator of the diffusion $Y$.

The function $\varphi_{\lambda}(\cdot)$ is characterised as the unique (up to a multiplicative constant) solution of (4.9) by demanding that $\varphi_{\lambda}(\cdot)$ is decreasing and satisfies some appropriate boundary conditions. The reader is referred to [35] for further details of the function $\varphi_{\lambda}(\cdot)$ and its relation to hitting times, as well as [15], Chap. 5, where examples of diffusions and associated functions $\varphi_{\lambda}(\cdot)$ are developed.

Proof of Proposition 4.11 Let us consider the hitting time

$$
\tau_{z}=\inf \left\{t \geq 0: \frac{s\left(Y_{t}\right)}{s(x)}=a\right\}=\inf \left\{t \geq 0: Y_{t}=s^{-1}(\operatorname{as}(x))\right\}
$$

for $a \geq 1$ and $z:=s^{-1}(a s(x)) \leq x$. The Laplace transform of $\tau_{z}$ has by [35] and [6, Chap. 2] the form

$$
\mathbb{E}_{x}\left[\exp \left(-\lambda \tau_{z}\right)\right]=\frac{\varphi_{\lambda}(x)}{\varphi_{\lambda}(z)} .
$$

It suffices to substitute $z=s^{-1}(a s(x))$ in (4.10) and use the resulting expression in (4.2).

\section{Appendix}

In this appendix, we recall from [24] a few facts about the natural conditions and the Parathasarathy conditions (P). For more details see [24].

Most of the properties which generally hold under the usual conditions remain valid under the natural conditions (for example, existence of càdlàg versions of martingales, the Doob-Meyer decomposition, the début theorem, etc.). Let us recall here the definition.

Definition A.1 A filtered probability space $\left(\Omega, \mathcal{F},\left(\mathcal{F}_{t}\right)_{t \geq 0}, \mathbb{P}\right)$ satisfies the natural conditions if the following two assumptions hold:

- The filtration $\left(\mathcal{F}_{t}\right)_{t \geq 0}$ is right-continuous.

- For all $t \geq 0$, and for every $\mathbb{P}$-negligible set $A \in \mathcal{F}_{t}$, all subsets of $A$ are contained in $\mathcal{F}_{0}$. 
This definition is slightly different from the definitions given in [5] and [24], but one can easily check that it is equivalent. The natural enlargement of a filtered probability space can be defined by using the following result.

Proposition A.2 ([24]) Let $\left(\Omega, \mathcal{F},\left(\mathcal{F}_{t}\right)_{t>0}, \mathbb{P}\right)$ be a filtered probability space. There exists a unique filtered probability space $\left(\Omega, \widetilde{\mathcal{F}},\left(\widetilde{\mathcal{F}}_{t}\right)_{t \geq 0}, \widetilde{\mathbb{P}}\right)$ (with the same set $\Omega$ ) such that:

- For all $t \geq 0, \widetilde{\mathcal{F}}_{t}$ contains $\mathcal{F}_{t}, \widetilde{\mathcal{F}}$ contains $\mathcal{F}$, and $\widetilde{\mathbb{P}}$ is an extension of $\mathbb{P}$.

- The space $\left(\Omega, \widetilde{\mathcal{F}},\left(\widetilde{\mathcal{F}}_{t}\right)_{t \geq 0}, \widetilde{\mathbb{P}}\right)$ satisfies the natural conditions.

- For any filtered probability space $\left(\Omega, \mathcal{F}^{\prime},\left(\mathcal{F}_{t}^{\prime}\right)_{t \geq 0}, \mathbb{P}^{\prime}\right)$ satisfying the two items above, $\mathcal{F}_{t}^{\prime}$ contains $\widetilde{\mathcal{F}}_{t}$ for all $t \geq 0, \mathcal{F}^{\prime}$ contains $\widetilde{\mathcal{F}}$, and $\mathbb{P}^{\prime}$ is an extension of $\widetilde{\mathbb{P}}$.

The space $\left(\Omega, \widetilde{\mathcal{F}},\left(\widetilde{\mathcal{F}}_{t}\right)_{t \geq 0}, \widetilde{\mathbb{P}}\right)$ is called the natural enlargement of $\left(\Omega, \mathcal{F},\left(\mathcal{F}_{t}\right)_{t \geq 0}, \mathbb{P}\right)$.

Intuitively, the natural enlargement of a filtered probability space is its smallest extension which satisfies the natural conditions. We also introduce a class of filtered measurable spaces $\left(\Omega, \mathcal{F},\left(\mathcal{F}_{t}\right)_{t \geq 0}\right)$ such that any consistent family $\left(\mathbb{Q}_{t}\right)_{t \geq 0}$ of probability measures, with $\mathbb{Q}_{t}$ defined on $\mathcal{F}_{t}$, can be extended to a probability measure $\mathbb{Q}$ defined on $\mathcal{F}$.

Definition A.3 Let $\left(\Omega, \mathcal{F},\left(\mathcal{F}_{t}\right)_{t \geq 0}\right)$ be a filtered measurable space such that $\mathcal{F}$ is the $\sigma$-algebra generated by $\mathcal{F}_{t}, t \geq 0$, i.e., $\mathcal{F}=\bigvee_{t \geq 0} \mathcal{F}_{t}$. We say that the $\operatorname{property}^{1}(P)$ holds if $\left(\mathcal{F}_{t}\right)_{t \geq 0}$ enjoys the following properties:

- For all $t \geq 0, \mathcal{F}_{t}$ is generated by a countable number of sets.

- For all $t \geq 0$, there exist a Polish space $\Omega_{t}$ and a surjective map $\pi_{t}$ from $\Omega$ to $\Omega_{t}$ such that $\mathcal{F}_{t}$ is the $\sigma$-algebra of the inverse images, by $\pi_{t}$, of Borel sets in $\Omega_{t}$, and such that for all $B \in \mathcal{F}_{t}, \omega \in \Omega, \pi_{t}(\omega) \in \pi_{t}(B)$ implies $\omega \in B$.

- If $\left(\omega_{n}\right)_{n \geq 0}$ is a sequence of elements of $\Omega$ such that for all $N \geq 0$,

$$
\bigcap_{n=0}^{N} A_{n}\left(\omega_{n}\right) \neq \emptyset,
$$

where $A_{n}\left(\omega_{n}\right)$ is the intersection of the sets in $\mathcal{F}_{n}$ containing $\omega_{n}$, then

$$
\bigcap_{n=0}^{\infty} A_{n}\left(\omega_{n}\right) \neq \emptyset .
$$

A fundamental example of a filtered measurable space $\left(\Omega, \mathcal{F},\left(\mathcal{F}_{t}\right)_{t \geq 0}\right)$ satisfying the property (P) can be constructed as follows. We take $\Omega$ to be equal to $\mathcal{C}\left(\mathbb{R}_{+}, \mathbb{R}^{d}\right)$, the space of continuous functions from $\mathbb{R}_{+}$to $\mathbb{R}^{d}$, or $\mathcal{D}\left(\mathbb{R}_{+}, \mathbb{R}^{d}\right)$, the space of càdlàg functions from $\mathbb{R}_{+}$to $\mathbb{R}^{d}$ (for some $d \geq 1$ ), and for $t \geq 0$, we define $\left(\mathcal{F}_{t}\right)_{t \geq 0}$ as the

${ }_{1}(\mathrm{P})$ stands for Parthasarathy since such conditions where introduced by him in [33]. 
natural filtration of the canonical process, and we set

$$
\mathcal{F}:=\bigvee_{t \geq 0} \mathcal{F}_{t}
$$

The combination of the property $(\mathrm{P})$ and the natural conditions gives the following notion.

Definition A.4 Let $\left(\Omega, \mathcal{F},\left(\mathcal{F}_{t}\right)_{t \geq 0}, \mathbb{P}\right)$ be a filtered probability space. We say that it satisfies the property $(N P)$ if it is the natural enlargement of a filtered probability space $\left(\Omega, \mathcal{F}^{0},\left(\mathcal{F}_{t}^{0}\right)_{t \geq 0}, \mathbb{P}^{0}\right)$ such that the filtered measurable space $\left(\Omega, \mathcal{F}^{0},\left(\mathcal{F}_{t}^{0}\right)_{t \geq 0}\right)$ enjoys property $(\mathrm{P})$.

In [24], the following result about extensions of probability measures is proved (in a slightly more general form).

Proposition A.5 Let $\left(\Omega, \mathcal{F},\left(\mathcal{F}_{t}\right)_{t \geq 0}, \mathbb{P}\right)$ be a filtered probability space satisfying property $(N P)$. Then the $\sigma$-algebra $\mathcal{F}$ is the $\sigma$-algebra generated by $\left(\mathcal{F}_{t}\right)_{t \geq 0}$, and for all consistent families of probability measures $\left(\mathbb{Q}_{t}\right)_{t \geq 0}$ such that $\mathbb{Q}_{t}$ is defined on $\mathcal{F}_{t}$ and is absolutely continuous with respect to the restriction of $\mathbb{P}$ to $\mathcal{F}_{t}$, there exists a unique probability measure $\mathbb{Q}$ on $\mathcal{F}$ which coincides with $\mathbb{Q}_{t}$ on $\mathcal{F}_{t}$ for all $t \geq 0$.

\section{References}

1. Azéma, J.: Quelques applications de la théorie générale des processus, I. Invent. Math. 18, 293-336 (1972)

2. Azéma, J., Meyer, P.-A., Yor, M.: Martingales relatives. In: Séminaire de Probabilités, XXVI. Lecture Notes in Math., vol. 1526, pp. 307-321. Springer, Berlin (1992)

3. Azéma, J., Yor, M.: Sur les zéros des martingales continues. In: Séminaire de Probabilités, XXVI. Lecture Notes in Math., vol. 1526, pp. 248-306. Springer, Berlin (1992)

4. Barlow, M.T.: Study of a filtration expanded to include an honest time. Z. Wahrscheinlichkeitstheor. Verw. Geb. 44, 307-324 (1978)

5. Bichteler, K.: Stochastic Integration with Jumps. Cambridge University Press, Cambridge (2002)

6. Borodin, A.N., Salminen, P.: Handbook of Brownian Motion-Facts and Formulae, 2nd edn. Birkhäuser, Basel (2002)

7. Cheridito, P., Nikeghbali, A., Platen, E.: Processes of the class sigma, last zero and draw-down processes. SIAM J. Financ. Math. 3, 280-303 (2012)

8. Chung, K.L.: Probabilistic approach in potential theory. Ann. Inst. Fourier 23, 313-322 (1973)

9. Coculescu, D., Nikeghbali, A.: Hazard processes and martingales hazard processes. Math. Finance 22, 519-537 (2012)

10. Cox, J.C.: Notes on option pricing I: Constant elasticity of variance diffusions. Stanford University, unpublished (1975)

11. Dellacherie, C., Maisonneuve, B., Meyer, P.-A.: Probabilités et Potentiel, Chapitres xvii-xxiv: Processus de Markov (fin). Compléments de Calcul Stochastique. Hermann, Paris (1992)

12. Duffie, D., Kan, R.: Multi-factor term structure models. Philos. Trans. R. Soc. Lond. Ser. A 347, 577-586 (1994)

13. Elliott, R.J., Jeanblanc, M., Yor, M.: On models of default risk. Math. Finance 10, 179-196 (2000)

14. Imkeller, P.: Random times at which insiders can have free lunches. Stoch. Stoch. Rep. 74, 465-487 (2002)

15. Jeanblanc, M., Yor, M., Chesney, M.: Mathematical Methods for Financial Markets. Springer, Berlin (2009) 
16. Jeulin, T.: Semi-martingales et Grossissements d'une Filtration. Lecture Notes in Math., vol. 833. Springer, Berlin (1980)

17. Kardaras, C., Kreher, D., Nikeghbali, A.: Strict local martigales and bubbles. Preprint (2011). http://arxiv.org/pdf/1108.4177.pdf

18. Kent, J.T.: Some probabilistic properties of Bessel functions. Ann. Probab. 6, 760-770 (1978)

19. Lévy, P.: Sur un problème de Marcinkiewicz. C. R. Acad. Sci. 208, 319-321 (1939). Errata p. 776

20. Li, L., Rutkowski, M.: Constructing random times through multiplicative systems. Preprint (2011). http://www.maths.usyd.edu.au/u/libol/Publications/Li_Rutkowski_SPA_Nov_13.pdf

21. Madan, D., Roynette, B., Yor, M.: From Black-Scholes formula to local times and last passage times for certain submartingales. Preprint (2008). http://hal.archives-ouvertes.fr/docs/00/26/18/68/PDF/ 2008-14.pdf

22. Madan, D., Roynette, B., Yor, M.: Option prices as probabilities. Finance Res. Lett. 26, $79-87$ (2008)

23. Mansuy, R., Yor, M.: Random Times and Enlargements of Filtrations in a Brownian Setting. Lecture Notes in Math., vol. 1873. Springer, Berlin (2006)

24. Najnudel, J., Nikeghbali, A.: A new kind of augmentation of filtrations. ESAIM Probab. Stat. 15, 39-57 (2011)

25. Najnudel, J., Nikeghbali, A.: On some universal $\sigma$-finite measures and some extensions of Doob's optional stopping theorem. Stoch. Process. Appl. 122, 1582-1600 (2012)

26. Najnudel, J., Nikeghbali, A.: On some properties of a universal $\sigma$-finite measure associated with a remarkable class of submartingales. Publ. Res. Inst. Math. Sci. 47, 911-936 (2011)

27. Najnudel, J., Roynette, B., Yor, M.: A Global View of Brownian Penalisations. MSJ Memoirs, vol. 19. Mathematical Society of Japan, Tokyo (2009)

28. Nikeghbali, A.: A class of remarkable submartingales. Stoch. Process. Appl. 116, 917-938 (2006)

29. Nikeghbali, A.: Some random times and martingales associated with $\operatorname{BES}_{0}(\delta)$ processes $(0<\delta<2)$. ALEA Lat. Am. J. Probab. Math. Stat. 2, 67-89 (2006)

30. Nikeghbali, A.: Enlargements of filtrations and path decompositions at non stopping times. Probab. Theory Relat. Fields 136, 524-540 (2006)

31. Nikeghbali, A.: An essay on the general theory of stochastic processes. Probab. Surv. 3, 345-412 (2006)

32. Nikeghbali, A., Yor, M.: Doob's maximal identity, multiplicative decompositions and enlargements of filtrations. Ill. J. Math. 50, 791-814 (2006)

33. Parthasarathy, K.-R.: Probability Measures on Metric Spaces. Academic Press, New York (1967)

34. Pitman, J., Yor, M.: Bessel processes and infinitely divisible laws. In: Williams, D. (ed.) Stochastic Integrals. Lecture Notes in Math., vol. 851, pp. 285-370. Springer, Berlin (1981)

35. Pitman, J., Yor, M.: Laplace transforms related to excursions of a one dimensional diffusion. Bernoulli 5, 249-255 (1999)

36. Platen, E.: A minimal financial market model. In: Kohlmann, M., Tang, S. (eds.) Mathematical Finance. Trends in Mathematics, pp. 293-301. Birkhäuser, Basel (2001)

37. Platen, E.: Arbitrage in continuous complete markets. Adv. Appl. Probab. 34, 540-558 (2002)

38. Platen, E., Heath, D.: A Benchmark Approach to Quantitative Finance. Springer, Berlin (2006)

39. Profeta, C., Roynette, B., Yor, M.: Option Prices as Probabilities. A New Look at Generalized BlackScholes Formulae. Springer, Berlin (2010)

40. Revuz, D., Yor, M.: Continuous Martingales and Brownian Motion, 3rd edn. Springer, Berlin (1999)

41. Yen, J.-Y., Yor, M.: Call option prices based on Bessel processes. Methodol. Comput. Appl. Probab. 13, 329-348 (2011)

42. Yor, M.: Grossissements d'une filtration et semi-martingales: théorèmes généraux. In: Lecture Notes in Math., vol. 649, pp. 61-69 (1978)

43. Yor, M.: Les inégalités de sous-martingales, comme conséquences de la relation de domination. Stochastics 3, 1-15 (1979) 